\title{
Inertia Certificates - Möglichkeiten zur Gewährleistung von ausreichender Momentanreserve im Verbundsystem
}

\author{
W. Gawlik@, J. Marchgraber, Y. Guo
}

\begin{abstract}
Die Momentanreserve ist von entscheidender Bedeutung für die Aufrechterhaltung der Frequenzstabilität in Drehstromsystemen. Derzeit wird sie fast ausschließlich von direkt angebundenen Synchrongeneratoren aufgebracht, deren träge rotierende Schwungmasse sich Drehzahländerungen widersetzt und dadurch Frequenzgradienten nach Ungleichgewichten zwischen Einspeisung elektrischer Leistung ins und Entnahme elektrischer Leistung aus dem Netz begrenzt. Es steht zu erwarten, dass die für das Verbundsystem verfügbare Schwungmasse im Zuge der steigenden Verbreitung von über Leistungselektronik angeschlossenen Einspeiseanlagen zukünftig abnehmen wird. Die daraus resultierenden höheren Frequenzgradienten stellen eine Gefährdung der Frequenzstabilität des Verbundsystems dar. Inertia Certificates sind eine Möglichkeit, den Beitrag von Erzeugungsanlagen zur Bereitstellung von Momentanreserve im Verbundsystem zu quantifizieren und der Momentanreserve einen Preis zuzuordnen, der ihrem Wert für den Systembetrieb entspricht. Sie ermöglichen einen fairen Ausgleich zwischen jenen Anlagen, die aufgrund ihrer physikalischen Wirkungsweise inhärent Momentanreserve bereitstellen, und solchen, die prinzipbedingt keine oder nur eine geringe Schwungmasse aufweisen. Außerdem können durch die Abtretung und Abgeltung der Bereitstellung von Momentanreserve kostenintensive Nachrüstungen von solchen schwungmassearmen und schwungmasselosen Einspeisern vermieden werden.
\end{abstract}

Schlüsselwörter: Inertia Certificates; Inertia-Zertifikate; Momentanreserve; Schwungmasse; Frequenzstabilität

Inertia certificates - possibilities to guarantee sufficient inertia in the power system.

The instantaneous reserve is of decisive importance for maintaining frequency stability in AC systems. Currently, it is almost exclusively provided by directly connected synchronous generators, whose inertia resists changes in rotational speed and, thus, limits frequency gradients after imbalances between the electrical power supply into and consumption from the network. It is to be expected that the rotating mass available for the power system will decrease in the course of the increasing spread of generation systems connected via power electronics. The resulting higher frequency gradients pose a threat to the frequency stability of the interconnected system. Inertia certificates are a way of quantifying the contribution of generating plants to the provision of instantaneous reserves in the interconnected system and of assigning the instantaneous reserve a price that corresponds to its value for system operation. They enable a fair balance between those systems that inherently provide instantaneous reserves due to their physical mode of operation and those that, due to their principle, have no or only low inertia. In addition, by assigning and compensating for the provision of the instantaneous reserve, the cost-intensive retrofitting of such low-inertia and no-inertia generators can be avoided.

Keywords: inertia certificates; Inertia-Zertifikate; inertia; inertial response; instantaneous reserve; frequency stability

Eingegangen am 8. Juli 2021, angenommen am 17. September 2021, online publiziert am 8. November 2021 (c) The Author(s) 2021

\section{Einleitung und Problemstellung}

Die Frequenz in einem Drehstromsystem ist keine systemtheoretische Zustandsgröße im engeren Sinn, weil sie nicht direkt mit einem physikalischen Energiespeicher verbunden ist. Das liegt daran, dass das Drehfeld, das im elektrischen Drehstrom als virtueller Welle die Leistungsübertragung vom Ort der Erzeugung zum Ort der Anwendung übernimmt, für sich genommen keine Trägheit besitzt. Dagegen sind die Drehzahlen von direkt an das Verbundsystem angeschlossenen rotierenden elektrischen Maschinen sehr wohl mit physikalischen Energiespeichern, nämlich den rotierenden Massen ihrer Wellensätze, verbunden und somit Zustandsgrößen, die sich nicht sprungartig ändern können. Weil die Drehzahlen von Synchronmaschinen wiederum sehr eng mit der Systemfrequenz gekoppelt sind, solange der Synchronismus nicht verlorengegangen ist, verhält sich die Systemfrequenz allerdings sehr wohl als Quasi-Zustandsgröße und wird sich bei begrenzter Anregung des Systems mit einem Leistungsungleichgewicht nicht sprungförmig ändern, sondern einen stetigen Verlauf aufweisen

Verwendet man vereinfachend das Modell eines Ein-MassenSchwingers [1] für die mittlere Systemfrequenz im Synchrongebiet, dann gilt als Zusammenhang zwischen der mittleren Systemfrequenz $f$ und der Leistungsbilanz zwischen eingespeister Leistung

Gawlik, Wolfgang, Fachbereich Management, Information, Technologie (MIT), Jade Hochschule Wilhelmshaven/Oldenburg/Elsfleth, Friedrich-Paffrath-Straße 101, 26389 Wilhelmshaven, Deutschland; Institut für Energiesysteme und Elektrische Antriebe, Fachbereich Energiesysteme und Netze, Technische Universität Wien, Gusshausstraße 25/E370-01, 1040 Wien, Österreich (E-Mail: wolfgang.gawlik@jade-hs.de); Marchgraber, Jürgen, Institut für Energiesysteme und Elektrische Antriebe, Fachbereich Energiesysteme und Netze, Technische Universität Wien, Wien, Österreich; Guo, Yi, Institut für Energiesysteme und Elektrische Antriebe, Fachbereich Energiesysteme und Netze, Technische Universität Wien, Wien, Österreich 


$$
P_{G}(t)-P_{L}(t)=2 \pi \cdot f \cdot J_{S y S} \cdot \frac{2 \pi d f}{d t}
$$

Dabei ist $J_{\text {sys }}$ die Summe der auf die synchrone Drehzahl bezogenen Trägheitsmomente der rotierenden Massen. Sehr häufig wird statt der Summe der Trägheitsmomente die Netzanlaufzeitkonstante $T_{A, S y s}$ verwendet, die sich aus dieser Summe und der Systemgröße $P_{\text {Sys }}$ sowie der Nennfrequenz $f_{n}$ als

$$
T_{A, \text { Sys }}=\frac{J_{\text {Sys }} \cdot\left(2 \pi f_{n}\right)^{2}}{P_{\text {Sys }}}
$$

ergibt. Als Momentanreserve wird diejenige Leistung bezeichnet, die während auftretender Leistungsungleichgewichte unmittelbar und unverzögert aus den rotierenden Massen entnommen oder in diese eingespeist wird. Für die Momentanreserve $P_{d f / d t}$ gilt also unter Verwendung des Erzeugerpfeilsystems

$$
P_{d f / d t}=-\frac{f}{f_{n}} \cdot T_{A, S y s} \cdot P_{S y s} \cdot \frac{d f / f_{n}}{d t}
$$

Als Systemgröße [2] wird in diesem Artikel und in diesen Gleichungen die aktuell versorgte Netzlast verwendet, d.h. es gilt $P_{\text {sys }}=P_{L}(t)$. Sie ist damit zeitabhängig und wegen der üblichen Lastgänge tagsüber und unter der Woche größer und nachts bzw. am Wochenende kleiner.

In die Netzanlaufzeitkonstante gehen alle Trägheitsmomente der individuellen rotierenden Massen $J_{i}$ ein. Es wird also

$$
J_{\text {Sys }}=\sum_{i} J_{i}
$$

verwendet. Mit den individuellen Trägheitsmomenten und unter Verwendung der individuellen Bemessungsleistungen $P_{n, i}$ der einzelnen Erzeugungsanlagen gilt außerdem

$$
T_{A, i}=\frac{J_{i} \cdot\left(2 \pi f_{n}\right)^{2}}{P_{n, i}}
$$

Auflösen nach $J_{i}$ und Einsetzen in die Bestimmungsgleichung der Netzanlaufzeitkonstante ergibt

$$
T_{A, S y s}=\frac{1}{P_{S y s}} \sum_{i} T_{A, i} \cdot P_{n, i}
$$

Die Netzanlaufzeitkonstante ist nach dieser Gleichung also die auf die Systemgröße bezogene Summe der mit ihrer jeweiligen Bemessungsleistung gewichteten individuellen Anlaufzeitkonstanten der einzelnen Erzeugeranlagen. Diese kann man z.B. näherungsweise mit $T_{A, i}=0 \mathrm{~s}$ für leistungselektronisch angebundene Photovoltaikanlagen, $T_{A, i}=5 \ldots 8 \mathrm{~s}$ für Dampfturbinenkraftwerke und $T_{A, i}=8 \ldots 10 \mathrm{~s}$ für Gasturbinenkraftwerke ansetzen. Für die Netzanlaufzeitkonstante kann man derzeit erfahrungsgemäß einen Wert von etwa $T_{A, \text { Sys }}=10 \ldots 12 \mathrm{~s}$ ansetzen

Dass die Netzanlaufzeitkonstante höher ist als die nach Bemessungsleistung gewichtete Summe der individuellen Anlaufzeitkonstanten der einzelnen Erzeugungsanlagen, liegt in erster Linie daran, dass diese Anlagen in den allerwenigsten Fällen dauerhaft mit ihrer Bemessungsleistung betrieben werden, sondern sich in einem mehr oder weniger ausgeprägten Teillastbetrieb befinden. Aber auch wenn sie im Teillastbetrieb nur mit einem Teil ihrer Bemessungsleistung zur Systemgröße beitragen, bringen sie ihre gesamte

$$
\sum_{i} P_{n, i} \geq P_{\text {Sys }}, \quad \text { also } \frac{1}{P_{\text {Sys }}} \sum_{i} P_{n, i} \geq 1
$$

kann und wird deswegen die Netzanlaufzeitkonstante im Normalfall größer sein als der mit den Bemessungsleistungen gewichtete Mittelwert der Anlaufzeitkonstanten der individuellen Anlagen. Außerdem kann und wird es auch auf der Verbraucherseite Anlagen geben, die schwungmassebehaftet sind und nicht über Frequenzumrichter, sondern direkt an das Netz angeschlossen sind. Dieser Aspekt wird bei der Definition der Netzanlaufzeitkonstanten nach Gleichung (2) allerdings nicht berücksichtigt.

Grundsätzlich kann man jedenfalls konstatieren, dass Erzeugungsanlagen mit großer Anlaufzeitkonstante und insbesondere solche, die sich im Teillastbetrieb befinden, überproportional zur Netzanlaufzeitkonstante und damit zur Bereitstellung von Momentanreserve beitragen, und sich diese Bereitstellung nicht in der Vergütung für die Energielieferung niederschlägt. Das ist insofern problematisch, als dass dann Beschaffungsmechanismen, die ggf. zukünftig zur Gewährleistung und Einhaltung einer mindestens erforderlichen Netzanlaufzeitkonstante einzelne neue und unkonventionelle Anbieter eines "Produktes" Momentanreserve ungerechterweise bevorzugen könnten, wenn nicht auch Erzeugungsanlagen, die ihren Anteil an der Bereitstellung von Momentanreserve übererfüllen, eine Vergütung dafür erhalten, die dem Wert dieser essentiellen Systemeigenschaft entspricht.

Inertia Certificates, so wie sie in diesem Artikel vorgestellt werden, sind eine Möglichkeit, den Beitrag von Erzeugungsanlagen zur Bereitstellung von Momentanreserve im Verbundsystem zu quantifizieren und der Momentanreserve einen Preis zuzuordnen, der ihrem Wert für den Systembetrieb entspricht.

\section{Methodik für Inertia Certificates}

Vor der Zuteilung bzw. Abgabe von Inertia Certificates [3] steht zunächst die Festlegung einer Mindest-Netzanlaufzeitkonstante

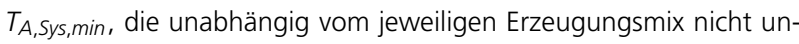
terschritten werden soll. Dieser Wert muss in Abstimmung aller Übertragungsnetzbetreiber im Synchrongebiet so gewählt werden, dass für die dimensionierenden Leistungsungleichgewichte und Systemzustände die dann auftretenden Frequenzgradienten und Frequenzabweichungen durch die vorgehaltenen Regelreserven (z.B. Primärregelung, also "Frequency Containment Reserve") beherrscht werden können. Für die vorgestellte Methodik spielt es keine ent-

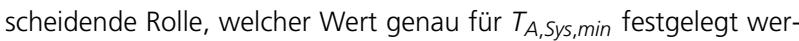
den wird. Für die folgenden Betrachtungen kann man z.B. von dem nicht ganz unplausiblen Wert von 5 s ausgehen [vgl. [5], S. 11], aber prinzipiell sind die Überlegungen für jeden und ggf. auch regional oder regelzonenspezifisch unterschiedliche Werte anwendbar.

Als nächstes wird für jede Erzeugungsanlage ein tatsächlicher individueller Wert der Anlaufzeitkonstante $T_{A, i}$ ermittelt. Diesen Wert kann man über die weiter oben aufgeführte Formel (5) aus dem vorhandenen Trägheitsmoment, der Nenndrehzahl und der Bemessungsleistung errechnen. Für das Trägheitsmoment kann man dabei auf vorhandene Daten bzw. Angaben des Herstellers zurückgreifen - die Berechnung erfolgt wie für jede andere Stabilitätsbetrachtung unter Berücksichtigung rotierender elektrischer Maschinen am Netz auch. Für bestimmte Arten von Einspeisern, z.B. Photovoltaikanlagen oder getriebelose Windkraftanlagen, die über Vollumrichter in das Netz speisen, wird das Ergebnis ohne zusätzliche Maßnahmen für Energiespeicher im Zwischenkreis und einer Anpassung der Umrichterregelung wohl $T_{A, i}=0$ s lauten. 


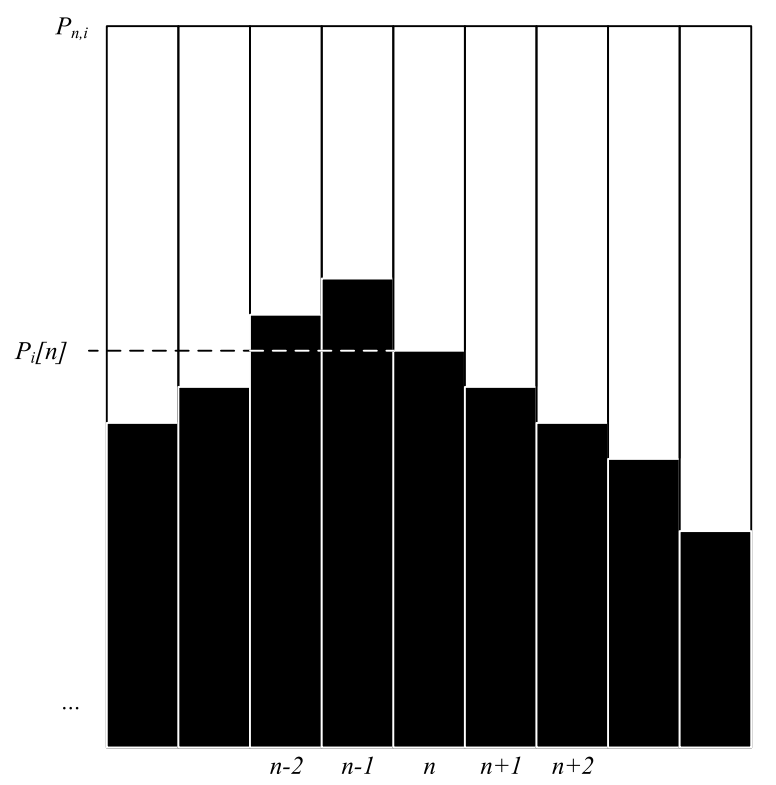

Abb. 1. Fahrplan einer Erzeugungsanlage in Form einer Abfolge von Arbeitspunkten

Die Methodik für Inertia Certificates basiert nun nicht auf einem Vergleich von $T_{A, \text { Sys, min }}$ mit $T_{A, i}$, sondern mit

$$
\hat{T}_{A, i}=T_{A, i} \cdot \frac{P_{n, i}}{P_{i}[n]}
$$

d.h. mit der Anlaufzeitkonstanten, die die ansonsten baugleiche Erzeugungsanlage aufweisen würde, wenn ihre dafür angesetzte Bemessungsleistung genau dem aktuellen Arbeitspunkt $P_{\mathrm{i}}[n]$ entsprechen würde. Die Abfolge dieser Arbeitspunkte ist im Rahmen der Erstellung und Prüfung der zugeordneten Fahrpläne der Anlage bekannt, üblicherweise in Form von Zeitreihen vergleichbar mit Abb. 1 mit z.B. einer 15-minütigen Abtastzeit. Weil im Regelfall $P_{\mathrm{i}}[n] \leq P_{n, i}$ sein wird, gilt also $\hat{T}_{A, i} \geq T_{A, i}$.

Mit diesen Überlegungen kann mal folgende Fallunterscheidungen treffen:

- $T_{A, \text { Sys, min }}<T_{A, i} \cdot P_{n, i} / P_{i}[n]$ : Für die Periode n übererfüllt die Anlage die Anforderungen zur Bereithaltung von Momentanreserve. Der überschüssige Anteil $T_{A, i} \cdot P_{n, i}-T_{A, \text { Sys, min }} \cdot P_{i}[n]>0$ kann als Inertia Certificate innerhalb dieser Periode mit anderen Erzeugungsanlagen gehandelt werden, die die Anforderungen nicht erfüllen. Dieser Fall ist in Abb. 2 und in Abb. 3, links oben dargestellt. Dabei entspricht der hellblau schraffierte Bereich gerade dem dunkelblau nicht-schraffierten Bereich, d.h. eine niedrige individuelle Anlaufzeitkonstante kann während eines Teillastbetriebes trotzdem mehr als ausreichend sein.

- $T_{A, \text { Sys,min }}=T_{A, i} \cdot P_{n, i} / P_{i}[n]$ : Für die Periode $n$ erfüllt die Anlage die Anforderungen zur Bereithaltung von Momentanreserve exakt. Das wird bis auf mehrere Stellen nach dem Komma genau wohl äußerst selten vorkommen. Aufgrund von Schwankungen und Messungenauigkeiten während einer Periode ist es deswegen empfehlenswert, einen Bereich um den Sollwert herum zu definieren, in dem die Bedingung als gerade erfüllt gewertet wird und weder Überschüsse noch Defizite ausgewiesen werden.

- $T_{A, \text { Sys,min }}>T_{A, i} \cdot P_{n, i} / P_{i}[n]$ : Für die Periode $n$ untererfüllt die Anlage die Anforderungen zur Bereithaltung von Momentanreserve. Der fehlende Anteil $T_{A, i} \cdot P_{n, i}-T_{A, S y s, \min } \cdot P_{i}[n]<0$ muss als Inertia Certificate innerhalb dieser Periode von anderen Erzeugungs-

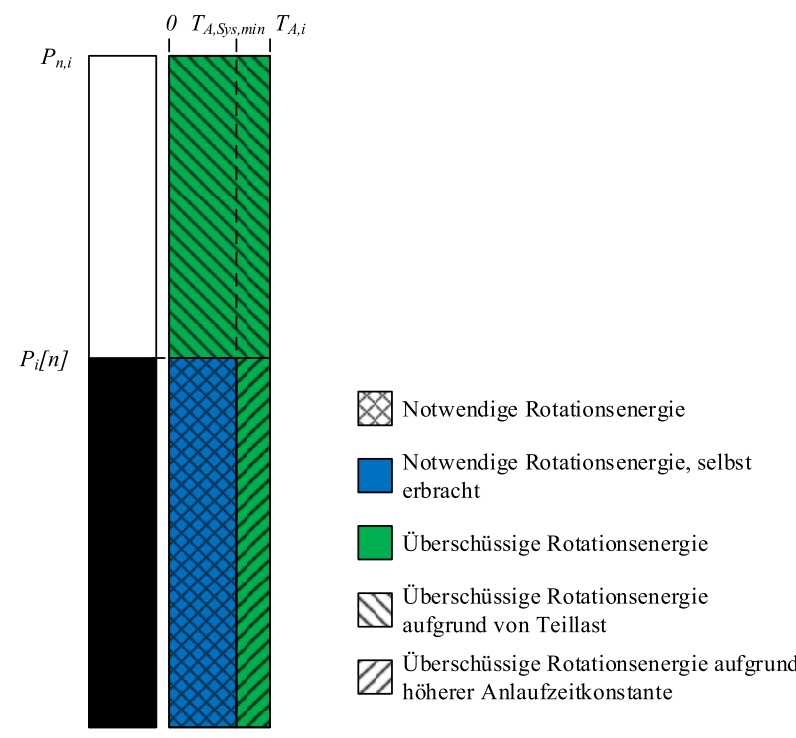

Abb. 2. Übererfüllung der Anforderungen zur Vorhaltung von Momentanreserve

anlagen beschafft werden, die diese Anforderungen übererfüllen. Dies gilt insbesondere natürlich für trägheitslose Erzeugungsanlagen (siehe auch Abb. 3 unten rechts).

Es sei an dieser Stelle noch angemerkt, dass die Inertia Certificates in der Form "Zeit multipliziert mit Leistung" die Einheit der Energie Joule (oder Ws) haben und letztlich auch vorgehaltene Rotationsenergie repräsentieren. Der Wert entspricht aber nicht dem Energieinhalt eines Energiespeichers, den man bei trägheitslosen Erzeugungsanlagen z.B. in Form von Hochleistungs-Kondensatoren im Gleichspannungs-Zwischenkreis nachrüsten müsste, denn jener ist wesentlich kleiner. Das liegt daran, dass auch bei einer konventionellen Schwungmasse nur jener Teil des Energieinhalts, der zwischen den Netzfrequenzen $47,5 \mathrm{~Hz}$ und 51,5 Hz mit dem System ausgetauscht wird, relevant ist. Dieser Teil entspricht in etwa $10 \%$ der gesamten Rotationsenergie bei Nenndrehzahl.

\section{Diskussion}

Die Art und Weise, wie ein Handel mit Inertia Certificates ablaufen wird, hängt sehr stark davon ab, auf welchen Wert die vorauszusetzende Mindest-Netzanlaufzeitkonstante $T_{A, S y s, \text { min }}$ festgelegt werden wird.

Solange diese Netzanlaufzeitkonstante jedenfalls durch die Erzeugungsanlagen gewährleistet werden kann, die inhärent Momentanreserve bereitstellen, wird der Preis von Inertia Certificates sehr gering sein und muss letztlich nur die Transaktionskosten abdecken, die den Anbietern von überschüssiger Momentanreserve bei einer Teilnahme an dem Handel entstehen. Es ist auch denkbar, dass in dieser Phase der Einführung von Inertia Certificates nur ein Monitoring der vorhandenen Momentanreserve durch die Übertragungsnetzbetreiber stattfindet, und der Handel mit Inertia Certificates noch ausgesetzt ist. Dieser Handel wird sinnvollerweise an den Energiehandel und die entsprechenden Fahrpläne gekoppelt sein, weil die geplanten Arbeitspunkte wie oben beschrieben wesentlicher Bestandteil bei der Ermittlung verfügbarer oder notwendiger Inertia Certificates sind. Nachdem alle Erzeugungsanlagen einer Bilanzgruppe zugeordnet sind, ermitteln auch die Bilanzgruppenverantwortlichen zusammen mit den aggregierten Fahrplänen für ihre Bilanzgruppe den "Momentanreserve-Fahrplan", damit er zusam- 

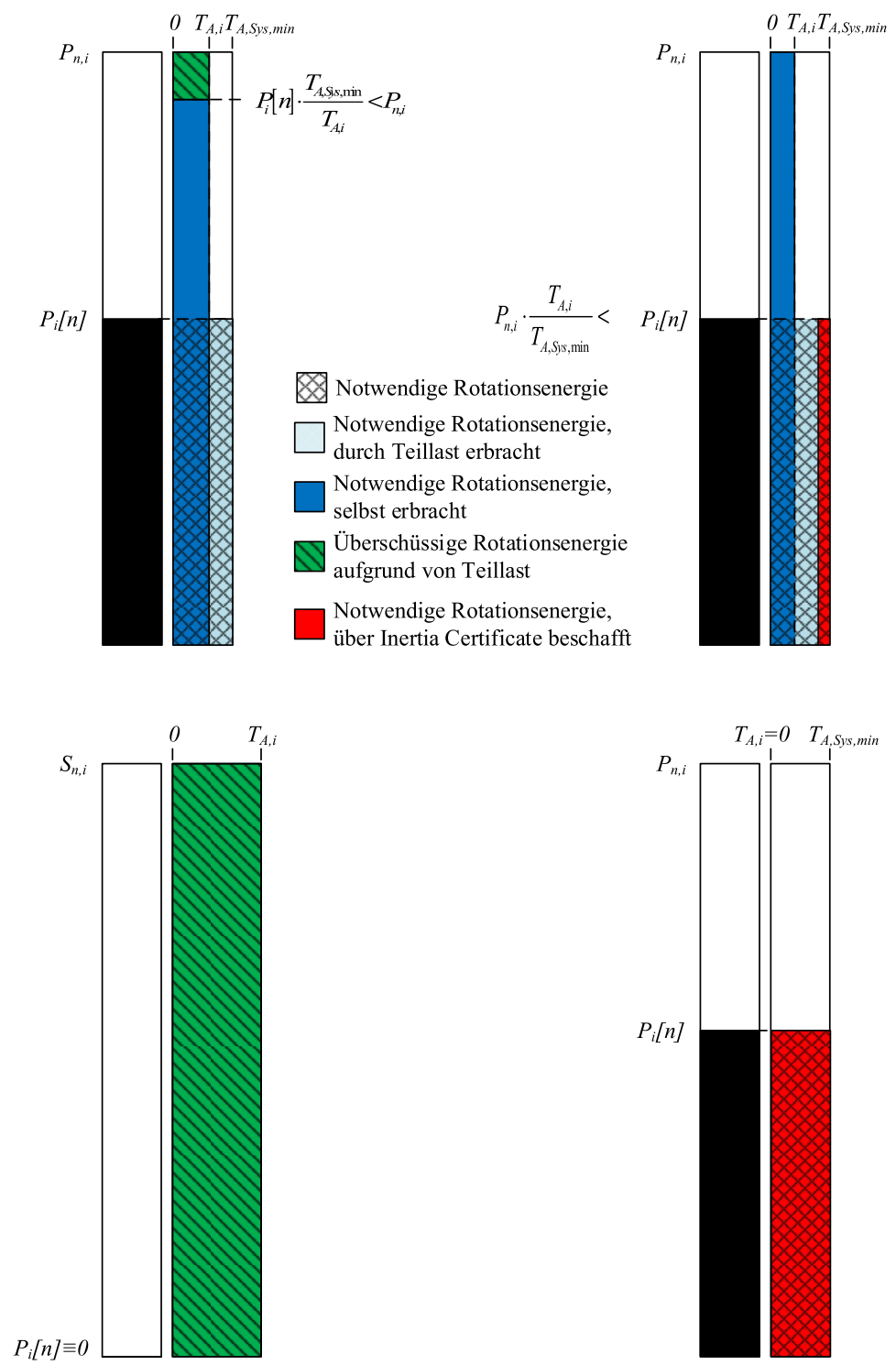

Abb. 3. Weitere mögliche Fälle für die Übererfüllung (oben links) bzw. Untererfüllung (oben rechts) der Anforderungen zur Erbringung von Momentanreserve, sowie trägheitslose Erzeugungsanlagen (unten rechts) und Schwungmasse-Bereitstellung z.B. durch Phasenschieber (unten links)

men mit allen anderen Fahrplänen für die gesamte Regelzone ausgewertet werden kann

Negative Preise für die Bereitstellung von Momentanreserve sind, anders als für die Energieeinspeisung, wegen

$$
T_{A, S y s, \min } \leq T_{A, S y s}=\frac{1}{P_{S y s}} \sum_{i} T_{A, i} \cdot P_{n, i}
$$

ausgeschlossen. Es sind derzeit keine Konstellationen denkbar, nach denen eine zu große Menge an Momentanreserve Nachteile für den Systembetrieb darstellen könnte. ${ }^{1}$ Der Preis ist also theoretisch mit 0 nach unten begrenzt.

${ }^{1}$ Nachdem sich aber in der Vergangenheit schon das eine oder andere Paradigma aufgelöst hat, wollen die Autor_innen nicht ausschließen, dass dies für Systemkonstellationen, für die ihnen heute noch die Phantasie fehlt, der Fall sein könnte. An der grundsätzlichen Methodik für Inertia Certificates ändert das aber nichts.
Umgekehrt wird der Preis für Inertia Certificates kaum die während der Periode, in der sie z.B. von trägheitslosen Erzeugungsanlagen beschafft werden müssen, erzielbaren Erlöse zur Energiebereitstellung überschreiten, aber natürlich diese Erlöse mindern. Dafür werden sich die Erlöse der Erzeugungsanlagen, die sich überproportional an der Bereitstellung von Momentanreserve beteiligen, durch den Handel mit Inertia Certificates erhöhen. Es scheint also absehbar, dass ein derartiges System den Preis der Momentanreserve, der derzeit nicht vorhanden ist, wesentlich besser an ihren wahren Wert anpassen wird.

Sobald der Preis für Inertia Certificates nämlich von Null verschieden wird, wird es für die Betreiberinnen von Erzeugungsanlagen, die sich aufgrund ihrer Bauart nicht ausreichend an der Bereitstellung von Momentanreserve beteiligen können, folgende vier Möglichkeiten geben:

- Keine oder geringere Beteiligung an der Energiebereitstellung („Abregeln“). Die Kosten für die Beschaffung von Inertia Certifi- 
cates erhöhen die Grenzkosten der jeweiligen Anlagen und „normalisieren" dadurch auch etwas die Merit-Order der Erzeugungsanlagen, indem der Anteil der betriebsabhängigen Kosten gesteigert wird. An dieser Stelle sei schon darauf hingewiesen, dass dies nicht bedeutet, dass die Kosten der Energieversorgung insgesamt steigen werden. Vielmehr bewirken die Inertia Certificates hier eine andere Verteilung der Erlöse unter Wahrung der notwendigen Systemeigenschaft "Vorhandensein von ausreichend Momentanreserve", während gleichzeitig Situationen zwar mit niedrigeren Energiekosten, aber Unterschreitung der mindestens benötigten Momentanreserve im System mit dem damit verbundenen Risiko höherer Folgekosten vermieden werden. Die Festlegung einer zu erreichenden Mindestanlaufzeit des Systems hat hier eine entscheidende Bedeutung.

- Erwerb von Inertia Certificates von Erzeugungsanlagen, die die Anforderungen zur Bereitstellung von Momentanreserve übererfüllen. Nachdem es sich dabei über den Verlauf mehrerer Abrechnungsperioden ( „Zeitscheiben") um eine variabel zusammengesetzte Gruppe von Erzeugungsanlagen handeln könnte, scheint es sinnvoll, hier Aggregatoren und Makler am Handel teilnehmen zu lassen, um das nachträgliche Clearing zu erleichtern. Durch die dabei anfallende Vergütung wird der Betrieb solcher Anlagen, die derzeit überwiegend zur Gewährleistung von Momentanreserve im System beitragen, wirtschaftlicher. Der Erlös reflektiert dann besser die Rolle dieser Anlagen zur Erhaltung der Systemintegrität.

- Erwerb von Inertia Certificates von Anlagen, die eigens zur Bereitstellung von Momentanreserve betrieben werden (z.B. Phasenschieber). Sobald ein marktbasierter Preis für die Bereitstellung von Inertia Certificates zur Verfügung steht, werden Geschäftsmodelle möglich, die auf der Bereitstellung von Momentanreserve basieren. Das kann z.B. die Umrüstung bestehender konventioneller Kraftwerke auf den Betrieb als Phasenschieber, ggf. mit erhöhter Schwungmasse, oder die Errichtung eigens dafür vorgesehener Anlagen sein, die im Rahmen eines multimodalen Betriebs auch für die Bereitstellung von Momentanreserve geeignet sind. Sobald Erfahrungswerte zur Ermittlung eines marktbasierten Preises für die Bereitstellung von Momentanreserve vorliegen, besteht auch eine erhöhte Planungssicherheit für solche Geschäftsmodelle.

- Aufrüstung der eigenen Erzeugungsanlage, um die Bereithaltung von Momentanreserve zu ermöglichen. Dazu können z.B. PVAnlagen mit einem (verhältnismäßig kleinen, siehe oben) Speicherelement Z.B. im Zwischenkreis eines Wechselrichters nachgerüstet und deren Regelalgorithmus so angepasst werden, dass eine Reaktion auf Frequenzgradienten im System erfolgt. Derartige Regelalgorithmen werden z.B. im Rahmen der "virtuellen Synchronmaschine" bzw. der "virtuellen Schwungmasse" oder "synthetischen Schwungmasse" seit längerem entwickelt [4], auch wenn es durch Einschränkungen bei der Stromtragfähigkeit der Ventile von Wechselrichtern und bei der regelungstechnischen Umsetzung zur Messung und Reaktion auf Frequenzgradienten ggf. zu Einschränkungen kommen kann. Die Wirtschaftlichkeit solcher Maßnahmen zur Nachrüstung bestehender oder Auslegung neuer Anlagen lässt sich jedenfalls besser beurteilen, wenn ein marktbasierter Preis für die Momentanreserve zur Verfügung steht, auch wenn hier grundsätzlich und unabhängig von der Methodik für "Inertia Certificates" die Fragestellung geklärt werden muss, wie "echt" die realisierte virtuelle Schwungmasse ist bzw. welcher Teil davon als Momentanreserve gewertet werden kann.

Die geographische Verteilung der Schwungmasse innerhalb eines Synchrongebietes und die Struktur des sie verbindenden elektri- schen Netzes haben entscheidende Auswirkungen auf mögliche Systemoszillationen einzelner Erzeugungseinheiten mit dem Rest des Netzes bzw. von Gruppen mehrerer Erzeugungseinheiten miteinander. Derartige niederfrequente Oszillationen mit Frequenzen im Bereich 0,1 bis $2 \mathrm{~Hz}$ können beispielsweise auch im kontinenaleuropäischen Verbundsystem beobachtet werden. Außerdem kann es während extremer Systemstörungen zum „System Split”, also dem Zerfallen des Synchrongebiets in mehrere vorübergehend getrennte Netzbereiche können, in denen anfänglich massive Unter- oder Überdeckung durch die vorher mit den anderen Netzbereichen ausgetauschte Wirkleistung und entsprechend große Frequenzgradienten auftreten können.

Für solche Fälle ist eine möglichst gleichmäßige, der Verteilung der Erzeugung und des Verbrauchs entsprechende Aufteilung der zur Verfügung stehenden Regelleistung und auch der wirksamen Schwungmasse wünschenswert. Die oben beschriebene Methodik der "Inertia Certificates" ist, wie oben beschrieben, an die Fahrpläne der Erzeugungseinheiten gekoppelt. Demzufolge bietet es sich auch an, einen Handel mit Inertia Certificates regional auf die jeweiligen Marktgebiete zu begrenzen und einen gebiete-übergreifenden Handel vergleichbar dem mit Regelreserven so abzuwickeln, dass keine zu großen überregionalen Ungleichgewichte entstehen, die sonst im Extremfall problematisch werden könnten. Je nach Ausprägung der Netzstrukturen und bekannter bzw. absehbarer Schwingungsneigung und anderer problematischer Dynamik könnten Regelzonenführer weitergehende Vorgaben machen und über Zu- bzw. Abschläge auf den Preis von Inertia Certificates deren Verteilung steuern, um die Tendenz zu Systemoszillationen und die Systemeigenschaften insgesamt positiv zu beeinflussen. Derartige Fragestellungen sollten und werden voraussichtlich zusammen mit den Betrachtungen zur Festlegung einer (ggf. regelzonen- oder regionalspezifischen) Mindest-Systemanlaufzeit angestellt werden müssen.

\section{Konklusion}

In diesem Artikel wird das Konzept der "Inertia Certificates" vorgestellt und die zugrundeliegenden physikalischen Überlegungen zur Schwungmasse in Drehstrom-Verbundsystemen dargelegt. Außerdem wird erläutert, wie ein marktbasierter Handel mit Inertia Certificates eine zu definierende mindestens notwendige Momentanreserve im Verbundsystem sicherstellen könnte.

Alternativ könnte man auch im Rahmen von Netzanschlussbedingungen von allen Erzeugungsanlagen verlangen, ein bestimmtes $\mathrm{Maß}$ an Momentanreserve bereitzuhalten. Für diejenigen Anlagen, die derartige Anforderungen derzeit übererfüllen, würde sich dadurch nichts verändern. Bestehende und neue Anlagen, die solche Anforderungen nicht erfüllen, müssten dann jedenfalls wie oben beschrieben nachgerüstet oder von vornherein passend dimensioniert und funktionell ausgerüstet sein. Inwieweit entsprechende Anschlussbedingungen die Integration von Anlagen zur Nutzung erneuerbarer Energieträger stärker belasten würde als zuzukaufende Zertifikate, lässt sich aktuell nicht vollständig abschätzen.

Ein marktbasierter Ansatz für den Handel mit Inertia Certificates würde jedenfalls bessere Voraussetzungen dafür schaffen, die Bereitstellung von Momentanreserve transparent und wirtschaftlich zu gestalten

Funding Note Open Access funding enabled and organized by Projekt DEAL.

Hinweis des Verlags Der Verlag bleibt in Hinblick auf geografische Zuordnungen und Gebietsbezeichnungen in veröffentlichten Karten und Institutsadressen neutral. 
Open Access Dieser Artikel wird unter der Creative Commons Namensnennung 4.0 International Lizenz veröffentlicht, welche die Nutzung, Vervielfältigung, Bearbeitung, Verbreitung und Wiedergabe in jeglichem Medium und Format erlaubt, sofern Sie den/die ursprünglichen Autor(en) und die Quele ordnungsgemäß nennen, einen Link zur Creative Commons Lizenz beifügen und angeben, ob Änderungen vorgenommen wurden. Die in diesem Artikel enthaltenen Bilder und sonstiges Drittmaterial unterliegen ebenfalls der genannten Creative Commons Lizenz, sofern sich aus der Abbildungslegende nichts anderes ergibt. Sofern das betreffende Material nicht unter der genannten Creative Commons Lizenz steht und die betreffende Handlung nicht nach gesetzlichen Vorschriften erlaubt ist, ist für die oben aufgeführten Weiterverwendungen des Materials die Einwilligung des jeweiligen Rechteinhabers einzuholen. Weitere Details zur Lizenz entnehmen Sie bitte der Lizenzinformation auf http://creativecommons.org/licenses/by/4.0/deed.de.

\section{Literatur}

1. Kundur, P. (1994): Power System Stability and Control. New York: McGraw-Hill.

\section{Autoren}

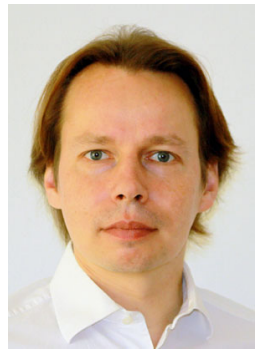

\section{Wolfgang Gawlik}

wurde 1971 in Landshut, Niederbayern, geboren. Er hat 1997 ein Studium der Elektrotechnik (Diplom) an der Friedrich-AlexanderUniversität Erlangen-Nürnberg abgeschlossen und wurde dort 2004 promoviert. Von 1997 bis 1999 war er Konstrukteur und stellvertretender Gruppenleiter Neukonstruktion im Siemens Generatorenwerk Erfurt. Von 2004 bis 2011 war er als Senior Key Expert System Dynamics und Senior Project Manager bei Siemens Power Technologies International in Erlangen beschäftigt. 2011 wurde er auf eine Professur für Energiesystemtechnik an die TU Wien berufen. Seit 2021 ist er Professor für Elektrische Energietechnik und Energiemanagement an der Jade Hochschule Wilhelmshaven.

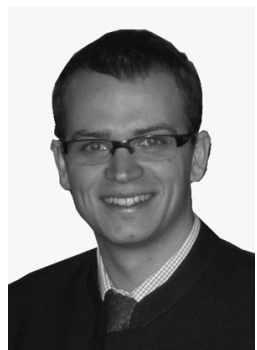

\section{Jürgen Marchgraber}

wurde 1988 in Linz, Oberösterreich, geboren. Er hat das Masterstudium Energietechnik an der Technischen Universität Wien im März 2014 abgeschlossen. Seit 2014 arbeitet er am Institut für Energiesysteme und Elektrische Antriebe der TU Wien als Universitätsassistent.
2. Tielens, P., Henneaux, P., Cole, S.: Penetration of renewables and reduction of synchronous inertia in the European power system - Analysis and solutions. Asset project, online, https://asset-ec.eu/wp-content/uploads/2018/12/EC_EUES_4NT_0631748_000_ 01 NTE.pdf. Abgerufen am 08.07.2021.

3. Gawlik, W., Lechner, A., Schürhuber, R. (2017): Inertia Certificates - Bedeutung und Wert von Momentanreserve für den Verbundnetzbetrieb; Vortrag: IEWT Internationale Energiewirtschaftstagung TU Wien, Wien; 15.02.2017-17.02.2017, in: Klimaziele 2050: Chance für einen Paradigmenwechsel? S. 1-8.

4. Dewenter, T., Werther, B., Hartmann, A. K., Beck, H-P. (2014): Optimierung des dynamischen Verhaltens netzstützender Anlagen am Beispiel der Virtuellen Synchronmaschine. In 13. Symposium Energieinnovation, Graz/Austria, 12.-14. Februar 2014.

5. ENTSO-E (2016): Frequency Stability Evaluation Criteria for the Synchronous Zone of Continental Europe - Requirements and impacting factors - RG-CE System Protection \& Dynamics Sub Group, online: https://www.entsoe.eu/Documents/SOC\%20documents/ RGCE_SPD_frequency_stability_criteria_v10.pdf. Abgerufen 18.08.2021

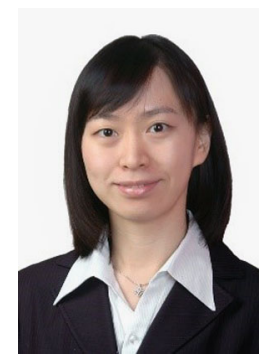

Yi Guo

hat $2018 \mathrm{ihr}$ Doktoratsstudium an der TU Wien abgeschlossen. Sie ist als Universitätsassistentin in Forschung und Lehre am Institut für Energiesysteme und Elektrische Antriebe der TU Wien tätig. Ihre Forschungsgebiete umfassen den Betrieb von Microgrids, Leistungs-/Frequenzregelung, Modellierung und Simulation von dynamischen Vorgängen im elektrischen Energiesystem und Netzwiederaufbau. 